\title{
Filigrane
}

Écoutes psychothérapiques

\section{Et s'il valait mieux se taire et attendre ?}

\section{Louise Larose-Cuddihy}

Volume 18, numéro 1, printemps 2009

Le corps. Sur le divan. Dans le fauteuil I

URI : https://id.erudit.org/iderudit/037718ar

DOI : https://doi.org/10.7202/037718ar

Aller au sommaire du numéro

Éditeur(s)

Revue Santé mentale au Québec

ISSN

1192-1412 (imprimé)

1911-4656 (numérique)

Découvrir la revue

Citer cet article

Larose-Cuddihy, L. (2009). Et s'il valait mieux se taire et attendre ? Filigrane, 18(1), 28-39. https://doi.org/10.7202/037718ar

\section{Résumé de l'article}

La pratique de la psychanalyse met l'analyste en contact avec son propre corps, touché par ce qui vient du corps de l'analysant. Celui-ci s'adresse à l'analyste en parlant de son corps, à partir de son corps. Réfléchissant sur quelques évocations cliniques, l'auteure se demande ce qui se passe entre l'analysant et l'analyste, quand le corps impose sa présence chez l'un comme chez l'autre, cela sans égard à l'organisation psychique qui prévaut chez l'analysant. Explorant alors le rapport entre le corps et la parole, elle s'interroge sur la façon d'aborder cliniquement ces différentes situations. Elle insiste, en conclusion, sur l'importance d'accéder à la parole comme issue à l'emprisonnement dans le corps, tout en précisant de quelle parole il s'agit. 


\title{
Et s'il valait mieux se taire et attendre?
}

\author{
louise larose-cuddihy
}

\begin{abstract}
La pratique de la psychanalyse met l'analyste en contact avec son propre corps, touché par ce qui vient du corps de l'analysant. Celui-ci s'adresse à l'analyste en parlant de son corps, à partir de son corps. Réfléchissant sur quelques évocations cliniques, l'auteure se demande ce qui se passe entre l'analysant et l'analyste, quand le corps impose sa présence chez l'un comme chez l'autre, cela sans égard à l'organisation psychique qui prévaut chez l'analysant. Explorant alors le rapport entre le corps et la parole, elle s'interroge sur la façon d'aborder cliniquement ces différentes situations. Elle insiste, en conclusion, sur l'importance d'accéder à la parole comme issue à l'emprisonnement dans le corps, tout en précisant de quelle parole il s'agit.
\end{abstract}

«l'enfance est un couteau dans la gorge»

« La scène de pendaison n'existe plus, elle n'est pas importante. Ce qui en reste, c'est l'idée d'étranglement qui empêche le cri et la parole de sortir. Et un désir profond de silence.»

Wajdi Mouawad

$\mathrm{E}$ ncore une fois, l'analyste se cale dans son fauteuil et, à son insu, son corps se raidit sous l'avalanche des paroles agressives que profère l'analysant. Le silence tombe mais non la détente; séance après séance, pendant des semaines, parfois des mois, le même scénario se répète et, insidieusement, un malaise physique s'installe chez l'analyste. D'autres fois, la description minutieuse et répétitive d'un problème physique crée chez l'analyste, comme en écho, une sorte de tension qui, elle aussi avec le temps, laisse des traces corporelles.

Que se passe-t-il, dans l'analyse, dans ces moments où le corps est pris à parti ?

D'où surgissent ces paroles qui véhiculent une charge telle que l'effet en est physique, que ce soit avec éclat ou insidieusement? Ces paroles, déjà mobilisées par le transfert, puisqu'elles sont adressées à l'analyste, semblent souvent communiquer davantage d'intensité que de représentations, telles des paroles-actions. L'analyste peut-il s'en servir de façon analytique, peut-il s'aider des manifestations non verbales pour mettre des mots sur la force qui les sous-tend? Quelle demande lui est alors adressée? 
À côté de ces moments où le corps est sollicité à travers la parole, existent ces autres temps où seul le corps parle, ceux où le discours n'est que description d'états corporels, de réactions à l'environnement physique; il y a encore toutes ces situations où le combat entre l'analyste et l'analysant est un combat à mort, pour la vie!

Réfléchir à ces questions implique un certain nombre de détours à travers les différents registres auxquels concourt un moment de travail analytique. L'origine des paroles alors entendues, comme celle de toutes les autres manifestations du corps, peut être considérée d'au moins deux façons : sous l'angle du surgissement de l'histoire singulière du sujet qui demande l'analyse, en tant qu'elle a façonné sa façon d'être, et sous celui du «fait psychique», tel qu'il est vécu dans la cure (Kahn, 2001, 1057). Les détours que nous prendrons, au cours de cette réflexion, seront surtout évocateurs comme ceux d'un voyageur porteur de questions, traversant de nombreux pays en quête d'éléments de réponse.

\section{«Tout cela me donne envie de vomir»}

Pour Tsunami, il est difficile de ne pas se sentir envahie par les odeurs du bureau, l'ordre ou le désordre qui y règne, les moindres mouvements de l'analyste. Il est tout autant difficile pour l'analyste de ne pas se sentir envahie ou agressée par la façon dont Tsunami l'aborde, s'inquiète d'elle, s'intéresse à son environnement, tel un débordement qui la met à nu, sans protection. Pourtant, dans un certain registre, une sorte de bonne volonté règne entre les deux. L'écart entre l'intention et le résultat n'est pas sans évoquer l'échec d'une harmonie, d'un accord entre la mère et l'enfant qui laisse alors ce dernier dans une détresse proche du traumatique.

Le magnifique travail d'élaboration que nous propose Piera Aulagnier, travail qui nous donne une théorie de la naissance de la pensée à partir du corps, semble particulièrement utile pour guider le travail clinique dans des moments où le corps occupe le premier plan. Son modèle de fonctionnement psychique est sur le mode d'un emprunt fait au corps. De la même façon que l'enfant nourri dans le déplaisir, à contretemps, a rejeté sa nourriture, l'adulte peut, dans des moments de souffrances émotives trop grandes, recourir à nouveau à cette forme de décharge expulsive, à la fois soulageante et souffrante, cela de façon réelle ou sous forme de déplacement.

Ce recours, comme la forme d'interaction évoquée plus haut, renvoie à un univers sans distance ni espace. Entre la mère et l'enfant, n'a pas eu lieu cette absence nécessaire à la naissance de la pensée, absence qui renvoie aux notions de tiers et de symbolique. La place alors imposée à l'analyste par le contre-transfert est celle de l'enfant mal pris dans un corps envahi par des décharges trop intenses, non liées par la parole; place due à l'incapacité de la mère à reconnaître une individualité différente de la sienne, dont il aurait fallu ensuite protéger et soutenir l'épanouissement. La présence de l'analyste, et encore plus sa parole, risquent toujours d'être éprouvées comme s'imposant agressivement; le moi-peau, sorte de 
filtre, ne joue pas son rôle de barrière de protection nécessaire. En même temps, cette expérience passée d'envahissement fait obstacle à la possibilité d'accéder à des relations médiatisées. L'analyste et l'analysant sont dans un corps à corps dont il faudra justement s'extraire peu à peu. C'est par petites touches, par absence et présence, en tâtonnant pour nommer l'éprouvé qui accompagne la description des malaises physiques, malaises qui tiennent souvent lieu d'un éprouvé, en laissant les dires de l'analysant demeurer au ras du corps, en acceptant l'étrangeté de ce qui se passe entre les deux, que peu à peu adviendra la parole.

Pour Mélancolia aussi, le sentiment d'écœurement est présent mais de façon différente, pas dans le réflexe d'expulsion mais plutôt dans le statu quo du malaise. Malaise qui laisse difficilement la place aux émotions et ne semble pas pour autant la libérer du poids de sa vie. La fatigue est devenue sa compagne inséparable et haïe tout comme ses préoccupations au sujet d'un surplus de poids dont son conjoint devrait la blâmer. Sans surprise, le diagnostic d'une maladie sérieuse mais contrôlable lui donne un nouvel espoir. Maintenant, elle est vraiment soignée. Malheureusement, l'identification à une mère rejetante, décédée trop tôt, ne cède pas si facilement. Être habitée par une autre pèse lourdement sur le cœur mais c'est le prix à payer pour maintenir un lien bien cruel mais qu'on espère tant transformer. Là aussi, le chemin est long tant pour l'analyste que pour l'analysant et, comme toujours, l'enjeu est transférentiel. Le corps malade ou ressenti comme tel évoque la remarque de Freud au sujet de la culpabilité inconsciente (Freud, 1923, 292): on se sent malade plutôt que coupable.

Les nombreuses situations où un corps étranger serait à expulser d'une manière ou d'une autre, sont sûrement présentes à la mémoire de tout clinicien. Elles ont en commun ce poids important vécu comme un élément toxique, nocif, étranger au corps propre, à soi-même, non pensé ou impensable en tant que faisant partie d'une histoire individuelle.

\section{Quand le besoin règne en maître}

Il est possible d'enrichir ce premier aperçu en puisant du côté de l'étayage. Déjà, le fait d'évoquer Aulagnier rappelle cette dimension d'abord si bien développée par Freud dans les Trois essais sur la théorie sexuelle (Freud, 1905, 102-107). Pour Aulagnier, reprenant Freud, c'est à travers l'expérience du plaisir, reliée à la satisfaction des besoins, qu'il est possible d'accéder éventuellement au registre du désir. À l'opposé, quand le déplaisir domine, s'installe l'agrippement à ce qui est, sans autre attente; parfois même, le désir de non-désir devient la seule solution à la souffrance qu'engendre l'accumulation de frustrations et l'envahissement du quantitatif.

Passer du besoin au désir, c'est ainsi passer d'un esclavage certain à un certain sentiment de liberté. Liberté de désirer, liberté accordée à l'objet du désir, plasticité du côté du choix d'objet. Mais comment, dans le travail clinique, offrir cette deuxième chance? Comment, par le travail du et sur le transfert, peut s'opérer un certain dégagement? Y aurait-il une façon de procéder spécifique à 
cette problématique ? Autrement dit, que faire de ces liens d'emprise, que faire de ces relations mères-filles qui constituent de véritables prisons dont on ne peut s'évader sans mourir, tout cela au prix de somatisations très graves ?

La difficulté à renoncer aux objets primaires, à faire le deuil de ce qu'ils n'ont pas été, semble souvent plonger l'analysant dans un corps à corps avec l'analyste, qui devient l'objet presque concret de cette quête de figures parentales idéalisées.

\section{La somatisation comme symptôme}

Sophie se retrouve en analyse à la suggestion de sa massothérapeute. Oui, elle croit qu'elle sera aidée de façon générale par cette démarche mais ne voit pas très bien comment ce travail peut améliorer son état physique. Maux de tête, tête vide, corps vécu comme origine de désastres multiples, toutes ces sources d'angoisse envahissent la jeune femme plutôt sceptique devant la démarche qu'elle souhaite entreprendre. À mesure qu'elle prend la parole, l'univers intérieur de Sophie prend forme et en même temps les imagos de son enfance; pendant longtemps, ses malaises physiques continuent cependant à s'imposer et à l'écraser tout en demeurant énigmatiques. Ses études universitaires la confrontent à la prise de parole et au regard des autres sur un discours porté par une femme et, par là même, convoquent aussi les symptômes qui ne semblent pas vouloir quitter la scène. Peu à peu pourtant, autour de l'image de la table, table de famille, table de travail, ses malaises somatiques deviennent, à ses yeux, équivalents les uns aux autres dans leur façon d'exprimer à la fois un interdit de penser le féminin et la preuve de l'échec d'une vie en tant que femme. Comment l'angoisse a-t-elle pu diminuer au point que Sophie puisse maintenant penser plutôt que de ressentir des malaises corporels?

Dans ce même registre, une autre analysante, Sylvie, vint à reconnaître, dans ses malaises physiques, une façon d'éviter ses désirs de femme que, pourtant, elle rêvait tant de réaliser. Chez elle, le travail se fit comme en parallèle: d'un côté de nombreuses séances où les symptômes physiques occupaient toute la scène; de l'autre, un espace d'expression de ses désirs plus intimes, timidement mis de l'avant. Périodiquement, les symptômes reprennent la place faisant penser à quel point le travail de résistance protège la satisfaction pulsionnelle qu'elle semble empêcher. Aller de l'avant, n'est-ce pas toujours aussi accepter de perdre?

Les barrières dressées par le symptôme, à la fois empêchement et réalisation inconsciente du désir, constituent le pain quotidien de notre travail. Faut-il cependant les considérer différemment, exigent-elles une autre approche, lorsqu'elles prennent la forme de diverses somatisations dont les descriptions remplissent souvent de très nombreuses séances?

\section{«Le moi est un moi corporel»}

Entre les évocations ci-dessus, il y a, malgré la singularité de chaque parcours et les différents registres dans lesquels il se déploie, suffisamment de similitudes pour qu'elles puissent être pensées sous un même angle. 
En effet, si le corps semble plus présent dans certaines analyses, ce n'est pas parce qu'il est absent dans les autres. Freud, Aulagnier, Mc Dougall, Fédida, Michel de M'Uzan, Rosolato, Nathalie Zaltzman, Christophe Dejours, pour n'en nommer que quelques-uns, sont parmi tous ces psychanalystes qui nous rappellent de différentes façons qu'il n'y a pas d'analyse qui ne se paie de «la livre de chair» (Zaltzman, 1974, 22), qui échappe au défi que la chair devienne parole et la parole chair (Monette, 2008). Parler vraiment, c'est toujours parler à partir du corps, dans le mouvement pulsionnel. Cependant, tout n'est pas si simple du passage du corps à la parole. «Le fait psychique, avant toute élucidation, écrit Laurence Kahn (2001, 1057), est un effet mais ce qu'il accomplit ne peut être compris dans le seul registre de ce qui s'éprouve. Freud, continue-t-elle, n'accorde aucune prévalence à l'intuition et à l'émotion comme outils d'une connaissance immédiate, pas plus qu'il n'élève le langage au rang d'unique fondement de la structure inconsciente. » Ce qui est rappelé ici est que l'essentiel de ce qui se passe dans l'analyse s'enracine dans le sexuel de l'analyste et de l'analysant. Dans ce sens, il est possible de considérer que la mise en mots est avant tout un agieren, en tant qu'expulsion de la charge libidinale, tant chez l'analyste que chez l'analysant.

D'où vient donc cette inquiétude de ne pas suffisamment tenir compte du corps dans l'analyse? D'où vient cette rumeur voulant que le divan, de par la «perte de vue» qu'il induit, constitue une négation du corps ? De quel corps parlons-nous? Il pourrait être tentant de réfléchir à ces questions en se centrant uniquement sur l'évocation de Tsunami au détriment des autres petits tableaux. Ou encore, à l'opposé, il pourrait paraître plus facile de mettre à l'écart l'histoire de Tsunami. On alléguerait alors, utilisant des catégories diagnostiques qui créent une distance confortable, qu'il vaut mieux recourir à son endroit à des «techniques » déjà éprouvées, réservant alors la méthode analytique pour des analysants davantage capables d'élaboration. Ce serait, me semble-t-il, oublier que la méthode analytique ne produit pas de découpage dans l'écoute, selon des catégories corps-parole; elle reçoit ce qui se présente comme effet du travail du psychisme, aux prises avec des forces contradictoires, à la recherche de sa propre satisfaction, travail déformant de toutes les manières possibles les manifestations du désir qui habite chacun.

«Le moi est un moi corporel» (Freud, 1923, 270), affirme Freud et, en note du même texte: «Il est une projection mentale de la surface du corps». Plus loin, Freud insiste : «Le moi conscient est avant tout un moi corps». Ces affirmations exigeraient de longs développements pour en déplier toute la richesse ainsi que les implications théoriques et cliniques. Il est possible ici de les utiliser de façon évocatrice, comme rappel du lien indissoluble entre le corps et la pensée médiatisés par la pulsion et ses représentants, pulsion définie, entre autres, par Freud «comme une mesure de l'exigence de travail qui est imposée à l'animique par suite de sa corrélation avec le corporel. » (Freud, 1915, 167)

Christophe Dejours a repris, d'une façon fort utile pour la clinique, l'essentiel de ces considérations sur le lien entre corps, pulsion et pensée. Pour lui, rejoignant en cela Aulagnier et Joyce Mc Dougall, 
«Tout se passe comme si la pensée à l'état naissant était, avant tout, une expression des états du corps et de leurs transformations. Le corps physiologique doit être progressivement l'objet d'une conquête subversive par le corps érotique.» (Dejours, 2005, 81)

C'est à travers l'investissement psychique du corps de l'enfant par la mère, s'exprimant dans les soins et dans la parole, que se produit cette subversion. Autrement dit, le corps doit être repris dans un travail de pensée, imposé par la butée que rencontre le mouvement pulsionnel dans sa recherche de satisfaction.

\section{Et s'il valait mieux se taire et attendre?}

Dans un même mouvement, il devient possible d'affirmer l'importance du corps dans l'analyse et la conviction que ce n'est qu'à travers la parole que devient accessible, tant à l'analyste qu'à l'analysant, tout le registre de son éprouvé, du nonverbal, du pré-verbal. Cette affirmation pourrait certes étonner. Le non-verbal, le pré-verbal, l'expression corporelle, le geste, les réactions physiques et les symptômes somatiques ne sont-ils pas omniprésents dans toute analyse ? Oui, sans doute, mais, tels quels, ils demeurent inutilisables, à cause de leur polysémie, de la déformation dont ils sont l'objet et, le plus souvent, dans la mesure où ils échappent à la mouvance du travail psychique ou en demeurent trop extérieurs. Certes, du côté de l'analyste, ils peuvent être des plus utiles pour lui signaler un mouvement contretranférentiel, mais là encore, dans la mesure où ils ne lui échappent pas.

Quel statut leur donner alors? On pourrait les considérer selon deux voies différentes, bien qu'elles soient dans la cure le plus souvent associées : en tant que symptôme ou en tant que début de figuration d'une parole qui cherche à trouver sa forme (Kahn, 2001).

Du côté du symptôme, la pensée de Dejours est encore ici fort stimulante: «L'origine et les conditions psychonévrotiques de la formation du symptôme, en terme de refoulement et de formation de compromis, sont de peu d'intérêt. » Il continue, soulignant que le symptôme, quelle que soit son origine, est d'abord pour l'analysant un moyen de ne pas penser et de ne pas dire. «... l'activité de penser liée au sexuel est désormais gênée ou empêchée par le symptôme» (Dejours, 2005, 76) et, poursuit-il, «... le pouvoir du corps de faire naître la pensée ne serait pas l'apanage d'un stade archaïque, mais le propre de tout fonctionnement psychique. »C'est ce qu'exprimait si bien cette analysante, après quelques années d'analyse, constatant que le problème physique auquel elle attribuait sa difficulté à vivre lui avait en fait servi d'écran, de prétexte pour ne pas explorer sa conviction de ne pouvoir vivre une relation amoureuse.

Il reste que de passer des sensations à la parole implique la création d'un espace psychique, suffisamment contenant pour que le risque des mots puisse être pris. Cela suppose assez de patience pour laisser place aux balbutiements, aux recherches tâtonnantes de mots, au dépassement du besoin d'être rassuré, à celui 
d'être dans une sorte de contact réel ou d'intimité palpable, concrète, avec l'analyste; cela suppose encore l'élaboration d'une capacité à tolérer le silence, le non-savoir. Le passage à la parole n'est jamais direct, même chez ceux qui utilisent facilement les mots. «Je n'ai plus envie, disait cet analysant, de parler pour parler, de seulement remplir l'espace pour me sentir proche de vous ou pour vous atteindre. Je sais de quoi je devrais vous parler mais je ne suis pas prêt. Je préfère me taire.» Dans le meilleur des cas, l'analysant a déjà fait l'expérience des mots de la mère qui, à l'époque du pré-verbal, ont su transformer les affects en sentiments (Aulagnier, 1975, 130-135), il a pu ensuite poursuivre ce travail pour luimême. Il y a alors prédominance de la parole mais une parole prise dans le transfert, c'est-à-dire dans le pulsionnel, dans le corps qui s'érotise par le transfert. Souvent cependant, selon l'expression de Sophie de Mijolla, le travail de l'analyste consiste à :

«Mettre les mots là où ils ont manqué, transformer un affect vécu comme une expérience innommable dont la réminiscence est devenue un danger permanent en une histoire dicible [.....] Car les mots reproportionnent et permettent au vécu, quel qu'il soit, non pas de s'effacer mais d'être lié, enserré dans un réseau causal exprimable au lieu d'être simplement subi. » $(1998,116)$

Mais cela, jamais trop tôt, jamais sur du non-médiatisé par une certaine parole. Laisser le temps au transfert de se développer. Laisser la répétition faire son œuvre, soutenir l'effort de mise en mots de l'analysant, sans précipitation, sans anxiété agissante, telle est bien la difficulté de l'analyste devant la multiplicité des manifestations liées au corps, parfois très inquiétantes, le plus souvent dérangeantes. Voilà bien aussi, ce qui a un effet sur le corps de l'analyste, pris dans sa propre organisation libidinale, et qui a à se contenir lui-même, cela en plus de recevoir la charge libidinale venant de l'analysant.

Pendant plusieurs séances, Ulysse joue avec ses doigts invitant l'analyste à l'aider à retrouver un jeu de son enfance. Quelques semaines plus tard, dans le prolongement d'une tempête transférentielle, il raconte un rêve sans résonance pour lui-même et reprend le jeu avec les doigts, l'accompagnant cette fois d'un commentaire qui lui donne accès, à travers une remarque de l'analyste, à tout un pan de son histoire actualisée dans la relation transférentielle. Cette lente mise en forme d'une expérience infantile traumatique, à travers une expression corporelle, un rêve, une parole, permet, dans le transfert, une reprise de l'événement passé qui favorise un nouvel investissement pulsionnel et un certain dégagement. Dégagement de ce dont déjà le corps parlait, à l'insu de l'analyste et de l'analysant, et qui ne pouvait être interprété sans un long travail de figuration.

Le risque pour l'analyste, quand le corps se fait trop présent, opaque, servant la résistance à l'élaboration de la pensée, serait de répéter le traumatisme d'origine. En effet, intervenir directement sur des manifestations corporelles, très souvent 
recrée, d'une part, la violence secondaire de l'interprétation (Aulagnier, 1975, 150) et, d'autre part, par l'excitation induite, une situation où le débordement agressif devient la seule issue possible.

\section{Et si nous avions peur du transfert?}

Est-il possible, à ce moment de cette réflexion, de faire l'hypothèse qu'une crainte inconsciente du transfert puisse être à l'œuvre par rapport au corps? (Mauger, Monette, 2000, 1418-1425) La lourdeur et la non-maîtrise (Zaltzman, 1996, 433) qu'implique la relation transférentielle pourrait parfois amener l'analyste à ignorer les manifestations trop dérangeantes du corps qui lui sont adressées, laissant ainsi dans l'ombre une part importante du transfert. Cette négation contribuerait peut-être à l'impression que la psychanalyse ignore le corps. Est-il possible aussi que la difficulté à tenir compte de la relation contre-transférentielle, inconsciente par définition, laisse trop souvent l'analyste dépassé par des déferlements somatiques perturbants?

Les remarques de certains analysants sont difficiles à recevoir. En plus des questions qui peuvent être entendues comme des interrogations sur l'état de l'analyste, renvoyant à différentes formes de la peur, de l'abandon ou du rejet, il y ces autres commentaires beaucoup plus directs qui déstabilisent souvent. Remarques diverses: vous êtes pâle, vous avez une curieuse couleur de cheveux, qu'avez-vous aux doigts, je n'avais pas remarqué tel ou tel détail de votre visage... À cela s'ajoutent souvent des demandes d'intervention sur leur état physique, un peu comme s'ils voulaient que l'analyste prenne soin concrètement d'eux-mêmes ou leur montre comment le faire. Le corps de l'analyste apparaît alors comme une possession de l'analysant, possession qui évoque l'exploration du corps de la mère comme façon de s'approprier son propre corps. La dépossession du corps propre de l'analyste s'ajoute à tant d'autres facettes de la relation contre-transférentielle.

En se plaçant du côté de l'analysant, il devient davantage possible de trouver les mots justes ou d'accepter le silence nécessaire pour soutenir sa démarche dans un grand respect de sa singularité, singularité qui vient de celle de son expérience du corps propre et qui façonne à la fois l'expression de sa pensée et de son fonctionnement inconscient. En agressant l'analyste par ses paroles, tel analysant le met à sa place d'enfant. Se taire, lui permettre de se calmer, de se retrouver en silence avec lui, d'éprouver le droit d'être là, est une façon de favoriser l'émergence éventuelle d'une parole pleine. Non, l'analyste ne comprend pas ce qui se passe, ne peut rien en dire, ne sait pas, car l'un n'est pas l'autre; le dedans et le dehors peuvent tout doucement s'installer.

Recevoir la souffrance de l'analysant aux prises avec des parties de lui-même laissées sans nom, qui échappent à l'investissement pulsionnel, implique pour l'analyste d'accepter d'être lui-même ébranlé dans sa propre identité. L'analyste ne peut éviter pour lui-même l'expérience de la régression souhaitée pour l'analysant, régression que Fédida décrit comme «l'imagination que se donne le vivant» 
du «courant continu de la vie ${ }^{1} »$. «Et semblable imagination, dit-il, fait place à des déplacements de lieux et des dyschronies qui concernent, dans la vie, les tendances sexuelles.» $(2000,6)$

Cette remarque de Fédida fait écho à l'exigence de non-maittrise imposée à l'analyste, non-maîtrise que favorise l'attention flottante et l'absence d'objectifs dans l'écoute de l'analysant.

Dans la mesure où l'analyste lui-même demeure en contact avec sa propre expérience de l'inconscient, on peut penser, selon les mots de Fédida, que l'analysant pourra, lui aussi, «... se donne[r] accès, en toute confiance, à sa vie psychique dans ce qu'elle a de plus angoissant» $(2000,7)$.

\section{La parole et le corps}

En conclusion, il serait utile de s'arrêter à nouveau sur le début de ce texte où est décrit une situation où le corps est pris à mal, tant pour l'analysant que pour l'analyste. Cette situation qui a servi d'introduction à une réflexion sur le rapport au corps dans l'analyse posait aussi la question de ce qui se passe, dans ces situations, pour que le corps de l'analyste soit ainsi atteint. Le texte ci-dessus apporte des éléments de réponse à cette difficile question, en mettant en évidence, les origines de la parole et de la pensée chez tout être humain ainsi que les implications transféro-contre-transférentielles de ces origines. L'accent est mis sur la nécessité de garder le travail analytique dans le registre de la parole, tout en reconnaissant la présence du corps dans toute analyse ainsi que les implications plus ou moins paralysantes de celui-ci.

Une dernière réflexion autour du corps et de la parole s'impose avant de clore ce texte. En effet, parler du corps en psychanalyse est avant tout ambiguë. Encore une fois, de quel corps s'agit-il? Le corps dont parle Freud, le corps humain, pourrait-on ajouter, est un corps pris dans le langage, dans le symbolique. «Le corps, ce charnier de signes» comme le dit si bien Baudrillard. L'analysant qui parle de son corps, parle de lui-même et de ses désirs. On pourrait d'ailleurs penser, que lorsque ce corps devient comme inanimé, mécanique, anatomique, il signale un effet de déshumanisation, il marque l'œuvre de la pulsion de mort.

Que demande un analysant quand il présente ses symptômes à l'analyste? De lui redonner la liberté d'aimer, l'espoir que la vie vaut la peine, de le remettre au centre de son histoire? Peu importe les diverses formulations qui cherchent à qualifier cette demande, profondément, elles tournent toujours autour de la possibilité d'aimer et d'être aimé. Le corps qui est mis de l'avant par l'analysant ou celui de l'analyste qui est l'objet de décharges non encore liées, sont des corps concernés par ce rapport au libidinal, qu'il soit nié ou trop envahissant.

«... l'analyste ne peut oublier que son corps est la scène en laquelle viennent se jouer les fantasmes du patient, ou encore le lieu imaginaire des désirs selon l'économie primitive d'un échange avec le corps des parents.... C'est précisément dans le 
rapport au silence que le corps présent au fantasme vient remplir les mots...» (Fédida, 1971, 114)

Dans le même texte déjà cité, Fédida s'interroge sur le corps dont il s'agit chez Freud. Il parle, à ce sujet, d'une recherche qui ne se laisse pas prendre à l'illusion d'un réalisme du corps, mais se fait plutôt «attentive à ce qui du corps réside dans les mots, s'inscrit dans les traces, reste gravé en mémoire au point de n'apparaître que comme réminiscence » (Fédida, 1971, 117).

Qu'en est-il alors du moi corporel? Reprenant le parcours de Freud, de la première théorie des pulsions à la seconde, Gantheret souligne comment, dans Le moi et le ça, «Le moi instance surgit [.....], dans l'ambiguïté d'être à la fois source et objet d'investissement. » $(1971,142)$. Ce moi, rappelle Gantheret, est en filiation avec le corps, avec l'organique, il est dérivé des sensations corporelles, il est projection mentale de la surface du corps. Cette projection introduit une distance, marque un chemin qui implique un travail de transformation, un passage du «corps réel au corps dans le fantasme». Et Gantheret d'ajouter:

«Dans la clinique comme dans la théorie, nous sommes donc amenés à considérer que le champ psychanalytique s'établit dans une coupure, qui laisse à l'un de ses bords le corps biologique, pour en assurer "de l'autre côté" la reprise dans un langage, dans un système de signes. » $(1971,146)$

La parole dont il est ici question a donc peu à voir avec un discours rationaliste. Elle est prise dans le mouvement pulsionnel, elle produit un effet sur l'interlocuteur et sur celui qui l'énonce ; «ainsi les mots sont-ils à double sens, infantile et sexuel, toujours prêt à se revivifier. » (Kahn 2001, 1067)

En même temps, s'impose la parole comme profondément humaine.

Pourtant, certains analysants ont tant de peine à parler, sont tellement immergés dans le corps et l'agir. Est-il vraiment légitime de vouloir quand même privilégier la cure par la parole ? Comment est-ce possible de faire advenir cette parole?

Dans l'avant-propos de son livre Moi, monde, mots, François Gantheret nous invite à revenir à Emmy von N... (Freud et Breuer, 1958, 48) et son incitation faite à Freud de ne pas «lui demander toujours d'où provient ceci ou cela, mais la laisser raconter ce qu'elle a à dire ». Pour Gantheret c'est de là que naît la psychanalyse dans la mesure où Freud s'est imposé le silence.

«Mise en suspens de l'activité fébrile de recherche des causes, de l'interrogation volontariste. Ne plus chercher, laisser la place, l'espace libre, et écouter ce qui peut alors se chuchoter, poindre et suspendre, l'inattendu, l'incongru. » $(1996,8)$ 
C'est la même injonction au silence que m'avait fait cet analysant, à un moment où $\mathrm{j}$ 'aurais pu penser qu'il aurait souhaité m'entendre. Tout dans sa posture, dans son langage non verbal, dans les remarques qui m'étaient adressées de façon provocatrice, invitait à parler, à nommer à sa place, et pourtant... « Taisez-vous », m'avait-il dit.

«Ce qu'on ne saisit peut-être pas assez, c'est comment bascule, dans ce moment-là, le statut de la parole. Jusque-là, elle est instrument, d'ailleurs plus ou moins fiable......[maintenant] C'est tout un autre monde qui s'impose: le monde des signes qui, d'instrumentaux qu'ils semblaient, deviennent la seule réalité présente, la réalité psychique. (Gantheret, 1996, 9-11)

« Taisez-vous.. » car tout reste à dire, à entendre, même si tant a déjà été dit. Car il y a une grande différence entre parler des choses de sa vie et, selon l'expression de Gantheret, «se laisser parler» (1996, 9-11).

Avoir accès à cette parole autre, étrangère à soi-même, même si parler demeure difficile, est une ouverture, une possibilité de dégagement inestimable.

C'est en effet dans cette parole

«... que se fait et se défait le corps, qu'il s'exproprie d'un vécu pour s'entendre en échos dans les fragments du rêve; c'est encore par elle que le corps se délie et redevient folie ou mythe, genèse et puissance des organes dans les apparences de la fleur, de l'animal, de la pierre et aussi des villes et des palais, des objets et des instruments.» (Fédida, 1972, 119)

Autrement dit, c'est par cette parole, issue d'un certain silence, dynamisée par le transfert, que le corps sort de sa concrétude, pour être repris dans le fantasme, pour être pensé, parlé, vécu libidinalement.

Et si, pour cela, il suffisait de s'en tenir à la méthode...?

louise larose-cuddihy 44 avenue glencoe, outremont, h3t $1 \mathrm{p} 9$ louise.cuddihy@sympatico.ca

\section{Note}

1. Les italiques sont des auteurs des citations. 


\section{Références}

Aulagnier, P., 1975, La violence de l'interprétation, Paris, PUF.

Dejours, C., 2005, Le corps, comme «exigence de travail» pour la pensée, in Psychopathologie de l'expérience du corps, Paris, Dunod, 63-107.

Fédida, P., 1971, L'anatomie dans la psychanalyse, Nouvelle Revue de psychanalyse, no 3, Paris, Gallimard, 109-127.

Fédida, P., 2000, Par où commence le corps humain. Retour sur la régression. Paris, PUF.

Freud, S., 1915, Trois essais sur la théorie sexuelle, Paris, Gallimard, 1987.

Freud, S., 1915, Pulsions et destins de pulsions, Paris, Euvres complètes, XIII, PUF, 1988.

Freud, S., 1920, Au-delà du principe de plaisir, Essais de psychanalyse, Paris, Payot, 1981.

Freud, S., 1923, Le moi et le ça, Paris, Euvres complètes, XVI, PUF, 1991.

Gantheret, F., 1971, Remarques sur la place et le statut du corps en psychanalyse, Nouvelle Revue de psychanalyse, $\mathrm{n}^{\circ}$ 3, Paris, Gallimard, 137-147.

Gantheret, F., 1996, Moi, monde, mots, Paris, Gallimard.

Kahn, L., 2001, L’hallucinatoire, la forme, la référence, Revue française de psychanalyse, LXV, 4, Paris, PUF, 10571075 .

Mauger, J., Monette, L., 2000, L'idéal transmis, Revue française de psychanalyse, LXIV, 5, Paris, PUF, 1391-1461.

Monette, L., 2008, Présentation lors du colloque de la SPM, texte non publié.

Zaltzman, N., 1974, Entre les mots inhabités et la livre de chair, Topique, n 14, Paris, EPI, 7-26.

Zaltzman, N, 1996, Apprendre: passer du contre au pour, Topique, n 61 , Paris, Dunod, 433-441. 